\title{
Spinal cord injury with central cord syndrome from surfing
}

\author{
Yaniv Steinfeld ${ }^{1} \cdot$ Yaniv Keren ${ }^{1} \cdot$ Elias Haddad $^{2}$
}

Received: 18 July 2017 / Revised: 1 January 2018 / Accepted: 3 January 2018

(c) International Spinal Cord Society 2018

\begin{abstract}
Introduction Central cord syndrome (CCS) is an injury to the center of the spinal cord. It is well known as a hyperextension injury, but it has never been described as a surfing injury. Our report describes this injury in detail.

Case presentation A 35-year-old male novice surfer presented to the emergency department with acute tetraplegia following falling off his surfboard and hitting sea floor at a shallow beach break. He was rescued by a fellow surfer while floating in the sea and unable to raise his head above sea level. Upon arrival at the hospital, tetraplegia and sensory deficits were noted. Radiological investigations showed advanced spinal stenosis at C4-6 levels. T2 magnetic resonance imaging (MRI) demonstrated myelopathy at C5-C6 level. He was diagnosed as having central cord syndrome, treated conservatively, and regained near full neurologic recovery after a month of rehabilitation.

Discussion Unique sport activities lead to unique injuries. It is important to accurately describe these injuries in order to create protective measures against them. Neurologic injuries in surfers are uncommon. With low-energy trauma, surfer's myelopathy is still the most common diagnosis, but central cord syndrome should be in the differential diagnosis.
\end{abstract}

\section{Introduction}

Central cord syndrome (CCS) is an injury to the center of the spinal cord, including white and gray matter. Schneider et al. [1] were the first to describe the syndrome in the 1950s as a result of severe hyperextension injury to the cord in older patients with a pre-existing hypertrophic cervical arthritis, causing tetraplegia characterized by a greater involvement of the upper limbs rather than the lower limbs, and a variable sensory loss below the level of injury. The upper motor tracts are located more centrally than the lower motor tracts. For this reason, the upper motor tracts are affected much more severely than the lower ones. Central cord syndrome is considered as a cervical spine hyperextension injury, usually in the elderly who suffer from degenerative narrowing of the spinal canal [2]. Hyperextension probably causes the cord to be compressed by osteophytes or by a buckled ligamentum flavum [3].

Yaniv Steinfeld

ya_steinfeld@rambam.health.gov.il

1 Orthopedic Surgery Division, Rambam Health Care Campus, Haifa, Israel

2 Spine Unit, Rambam Health Care Campus, Haifa, Israel
Surfers injuries are rarely neurological. Most surfing injuries are lacerations, strains, sprains, and fractures [4]. Among neurologic injuries, surfer's myelopathy, a nontraumatic paraparesis, is the most common neurologic entity described in the literature, with 64 cases reported so far in the literature $[5,6]$. This non-traumatic entity is almost always described in a young, healthy, novice surfer with no pre-existent spinal disease. A vascular origin, presumably arterial insufficiency related to cervical spine hyperextension, is suspected as the underlying cause for this condition. Usually, all patients with incomplete injuries improve. More than $50 \%$ of patients improve and become ambulatory. Once neurological improvement has stopped, one should not expect further improvement in symptoms [7].

Another possible etiology for CCS is that surfers, while paddling their boards in a hyperextended position or attempting to stand up on the boards, perform the Valsalva maneuver, which may increase intraspinal pressure [8]. Increased intraspinal pressure leads to increased retrograde venous pressure in the epidural venous plexuses secondary to obstruction of the inferior vena cava. Why novice surfers are prone to this kind of injury has been postulated to that their neck muscles are not well developed as they are inexperienced surfers. The underdeveloped muscles can not protect the spine while performing numerous activities of neck hyperextension. It is not the sole etiology, but it has an added effect [6]. 
Surfer's myelopathy, although first described in surfers, was also described as a result of other hyperextension activities. For example, a 16-year-old female who became symptomatic after performing a series of back handsprings during gymnastics practice [9]. There is another case report describing this condition in a 7-year-old girl who became symptomatic 1 day after a cheerleading workout [10]. Another case was reported in a 51-year-old demolition worker [11].

The differential diagnosis of acute low-energy cord injury in a surfer should include surfer's myelopathy as the diagnosis of choice. Central cord syndrome is usually not suspected as a surfing injury, especially when the patient is young and previously healthy. This entity was never reported earlier in the literature as a surfing injury.

\section{Case presentation}

A 35-year-old previously healthy novice surfer was admitted to the emergency department with acute tetraplegia following falling off his surfboard and hitting sea floor at a shallow beach break. He was rescued by a fellow surfer while floating in the sea unable to raise his head above sea level. He is physically active to a moderate degree. He is a physiotherapist and notes that during his work he is active and uses his strength. Besides surfing, he runs on a regular basis and medium distances once a week. This incidence occurred on his 12th surfing session.

At presentation, he was stable from a hemodynamic and respiratory standpoint, vital signs within the normal range, and fully conscious. His Glasgow Coma Scale (GCS) was 15. He could only remember the time he was floating, unable to move and waiting for rescue.

He presented with sharp neuropathic pain in his arms, mostly the right side, aggravated by light touch, severe weakness, and inability to ambulate with more than half key muscle functions with a muscle grade less than three in both upper and lower limbs. American Spinal Injury Association Impairment Scale (AIS) grade was C. His motor and sensory function according to the International Standards for Neurological Classification for Spinal Cord Injury (ISNCSCI) was not recorded at his initial presentation. Rectal examination was performed by the general surgeon in the Emergency Room (ER) and was described as normal. In a second physical examination, a few hours after admission more than half of key muscle functions in the lower limbs were more than three. Yet still less than half of the key muscle functions in the upper limbs were less than three. He had allodynia and a burning sensation, mostly in his right arm. Association Impairment Scale grade was D. His motor and sensory function according to the ISNCSCI was pin prick 91 , light touch 88 , upper limb motor 27 , and

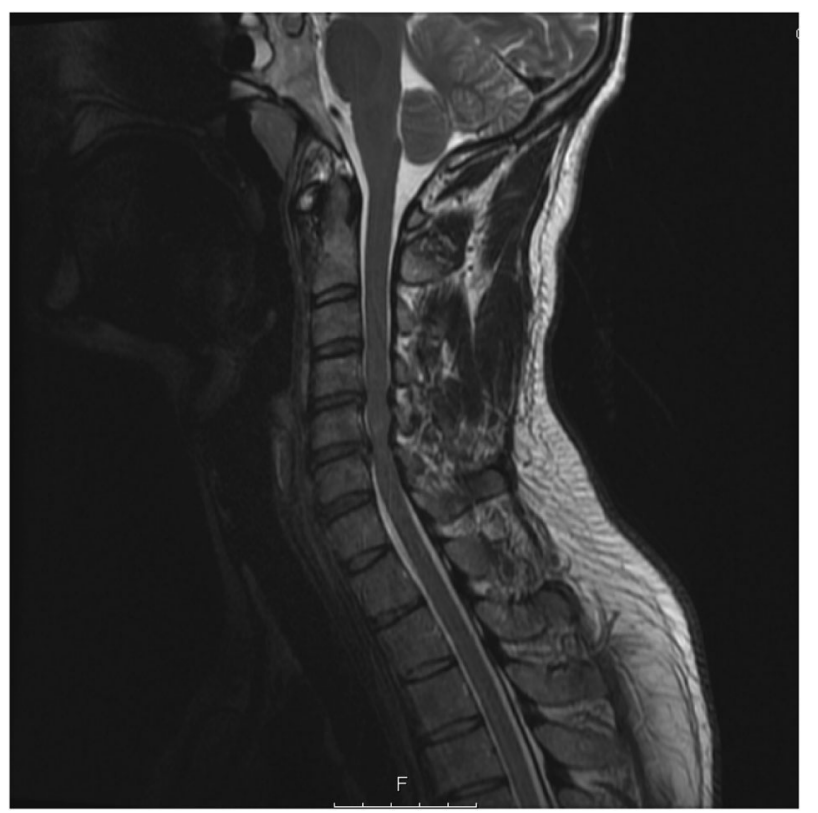

Fig. 1 Sagittal T2 weighted image demonstrating central stenosis at C4-C5 level and C5-C6 level

lower limb motor 26. Inspection of the spine was normal with no mid-line tenderness and no visible deformation or wound laceration. Deep tendon reflexes were brisk, with bilateral sustained clonus. Rectal examination was normal with normal anal sphincter tonicity, normal voluntary contraction, and normal perianal sensation.

Plain X-ray of his spine was normal with no fractures, deformation, or degenerative signs. Computed tomography (CT) scan revealed normal cranium, brain, chest, and abdomen. Cervical spine CT scan revealed no signs of hematoma, fracture, or soft tissue swelling. A cervical spine MRI was performed, which revealed disc bulges at $\mathrm{C} 4-\mathrm{C} 5$ and C5-C6 levels, loss of disc space height, and narrowing of the spinal canal, causing central spinal stenosis (Fig. 1).

Six hours after his arrival, he continued to show improvement with more than half of key muscle functions with muscle grade of more than three. The area of allodynia diminished and was now only present distal to his right elbow. He was admitted for hospitalization in the spinal surgery unit and received adequate analgesia and anti coagulation. Two days after his hospitalization, we began treating him with physical therapy for his upper limbs strength and physiotherapy for his walking imbalance.

His symptoms gradually improved during his hospitalization. The burning sensation and allodynia lessened, and a general improvement in muscle strength was noted. Three days after his arrival, he was able to walk using a simple walker, and more importantly for him, he was able to gently shake one's hand.

A detailed neurologic examination was made prior to his discharge to a rehabilitation facility and showed sensory and 
Table 1 Differential diagnosis of surfer's myelopathy and CCS

\begin{tabular}{lll}
\hline & $\begin{array}{l}\text { Surfer's } \\
\text { myelopathy }\end{array}$ & $\begin{array}{l}\text { Central cord } \\
\text { syndrome }\end{array}$ \\
\hline $\begin{array}{l}\text { Distinct injury } \\
\text { Pre-existing spinal } \\
\text { abnormality }\end{array}$ & No & Usual \\
$\begin{array}{l}\text { Prior symptoms } \\
\begin{array}{l}\text { Development of } \\
\text { symptoms }\end{array}\end{array}$ & No & Usual \\
Prognosis & Gradual & No \\
\hline
\end{tabular}

motor impairment at C5-T1, and general weakness in the lumbar region. His motor impairment was higher at his left side. Association Impairment Scale grade was D. His motor and sensory function according to the ISNCSCI was pin prick 105, light touch 98, upper limb motor 35, and lower limb motor 40 .

He was discharged and admitted to a Rehabilitation Department in another hospital for 3 weeks. During the hospitalization, he was treated by a multidisciplinary team consisting of rehabilitation physicians, physiotherapists, and occupational therapists. After physical therapy, there was slight improvement in hypersthesia in the upper limbs. Exercises were performed to strengthen the hands, increase the range of movement in the joints of the arm and palms, and exercise precise hand movements. In addition, he underwent basic and instrumental training. In light of his lack of balance, he underwent exercises to improve walking quality and balance. On a follow-up examination after he was discharged from the Rehabilitation Department, he showed great improvement, with almost all of his symptoms diminished. Some neurologic deficits were still present but he had almost no subjective complaints. He was AIS D with pin prick sensation 106 (baseline 105), light touch sensation 102 (98), upper motor strength 40 (35), and lower motor strength 50 (40).

The last time he came to the outpatient clinic, 3 months after his injury, he still had not returned to work. He suffered from imbalances in walking. In addition, he complained of cramps in the leg muscles and parasthesia in his hands in areas where he suffered from allodynia, suitable for spasticity neuropathic pain. He was offered surgical decompression of the cervical spine stenosis but he did not return to follow-up.

The patient was informed of our intention to publish his case and signed a consent form for case reports.

\section{Discussion}

Surfing is a popular sport around the world. That sport can lead to injuries. It is important to accurately describe these injuries in order to create protective measures against them. Neurologic injuries in surfers are uncommon. Review of the literature reveals surfer's myelopathy as a known and well-established surfing injury. Central cord syndrome, on the other hand, is mainly described as a phenomenon in the elderly with degenerative spine changes. It is not the usual suspect in case of a surfing injury. Table 1 outlines the differences between these two different entities.

Central cord syndrome can also be seen in younger individuals explained by congenital spinal stenosis [12, 13]. As seen in Fig. 1, our patient is suffering from some degree of congenital stenosis. When measuring his Torg and Pavlov canal-to-body ratio [14], the $a / b$ ratio is well $<0.80$. Our conclusion is that the case is the result of combination of trauma and congenital stenosis.

Novice surfers should be warned for the potential neurologic injuries from surfing. Gradual and cautious elevation of surfing endurance and adjusting surfing level to sea condition are important precautions that should always be advised.

\section{Compliance with ethical standards}

Conflict of interest The authors declare that they have no conflict of interest.

\section{References}

1. Schneider RC, Thompson JM, Bebin J. The syndrome of acute central cervical spinal cord injury. J Neurol Neurosurg Psychiatry. 1958;21:216-27.

2. Fehlings MG, Tetreault L, Nater A, Choma T, Harrop J, Mroz T, et al. The aging of the global population: the changing epidemiology of disease and spinal disorders. Neurosurgery. 2015;77: S1-5.

3. Quencer RM, Bunge RP, Egnor M, Green BA, Puckett W, Naidich TP, et al. Acute traumatic central cord syndrome: MRIpathological correlations. Neuroradiology. 1992;34:85-94.

4. Nathanson A, Haynes P, Galanis D. Surfing injuries. Am J Emerg Med. 2002;20:155-60.

5. Freedman BA, Malone DG, Rasmussen PA, Cage JM, Benzel EC. Surfer's myelopathy: a rare form of spinal cord infarction in novice surfers: a systematic review. Neurosurgery. 2016;78:602-11.

6. Thompson TP, Pearce J, Chang G, Madamba J. Surfer's myelopathy. Spine. 2004;29:E353-6.

7. Bosch A, Stauffer ES, Nickel VL. Incomplete traumatic quadriplegia: a ten-year review. JAMA. 1971;216:473.

8. Chung HY, Sun SF, Wang JL, Lai PH, Hwang CW. Nontraumatic anterior spinal cord infarction in a novice surfer: a case report. J Neurol Sci. 2011;302:118-20.

9. Albuja AC, Qaiser S, Lightner DD, Raslau FD, Zafar MS, Bernard PA, et al. Surfer's myelopathy without surfing: a report of two pediatric patients. Spinal Cord Ser Cases. 2017;3:17008.

10. Wadia S, Padmanabhan P, Moeller K, Rominger A. Pediatric surfer's myelopathy. J Emerg Med. 2015;49:e143-5. 
11. Maharaj MM, Phan K, Hariswamy S, Rao PJ. Surfer's myelopathy: a rare presentation in a non-surfing setting and review of the literature. J Spine Surg. 2016;2:222-6.

12. Kessler JT. Congenital narrowing of the cervical spinal canal. J Neurol Neurosurg Psychiatry. 1975;38:1218-24.
13. Verbiest $\mathrm{H}$. A radicular syndrome from developmental narrowing of the lumbar vertebral canal. J Bone Jt Surg Br. 1954;36-B:230-7.

14. Pavlov H, Torg JS, Robie B, Jahre C. Cervical spinal stenosis: determination with vertebral body ratio method. Radiology. 1987; $164: 771-5$. 